\author{
HERAUSGEBER: \\ Geh. San.-Rat Prof. Dr. Schwalbe \\ Berlin-Charlottenburg, Schlüterstr. 53 \\ VERLAG: \\ GEORG THIEME • LEIPZIG \\ AntonstraBe 15
}

\section{Der Sekundenherztod.}

Von Prof. H. E. Hering in Köln.

Der Name Sekundenherztod soll einen Vorgang zum Ausdruck bringen, der innerhalb von Sekunden durch Versagen des Herzens zum Tode führt. Die Bezeichnung Herztod ist geläufig, und es ist auch lange bekannt, daß der Herztod ein allmählicher oder ein plötzlicher sein kann. . Zur Charakterisierung seiner Plötzlichkeit dient seit Alters her der Ausdruck Herzschlag. Wozu also noch die Bezeichnung Sekundenherztod? Ich ${ }^{1}$ ) habe diesen Ausdruck eingeführt, um dem Begriff der Plötzlichkeit des Todes eine möglichst präzise Zeitbestimmung zu geben, da er in der Literatur nichts weniger als scharf gefalit wird. Das gilt nicht nur für diesen Begriff, sondern lauch für den Ausdruck Herzschlag, denn letzterer wird gebraucht, ob der Tod Sekunden, Minuten, Stunden oder selbst Tage nach dem Herzschlage erfolgte.

Worin liegt nun der Nutzen und der Fortschritt der neuen Bezeichnungsweise? Nicht allein darin, daß in Zukunft überhaupt auf den Zeitraum genauer geachtet werden wird, innerhalb dessen ein Individuum plötzlich stirbt, sondern auch darin, daß speziell beim plötzlichen Herztode aus der Kenntnis, daß der Herztod innerhalb von Sekunden erfolgte, für die Erkennung der Art und $W$ eise, in der das Herz versagt hat, ein wichtiger Anhaltspunkt gewonnen werden kann. Denn, wie ich kürzlich hervorgehoben habe: Der vom Arzte beobachtete Sekundenherztod, für dessen Plötzlichkeit die Sektion keinen entsprechenden Befund ergibt, läßt sich auf Grund der experimentellen Erfahrungen am Säugetierherzen nur durch Herzkammerflimmern erklären.

Der Sekundenherztod läßt also an eine besondere Art des Herztodes denken, den Tod durch Herzkammerflimmern; eine Art, die dem ärztlichen Denken noch garnicht geläufig ist.

Im Jahre 1904 hat E. Ehrnrooth ${ }^{2}$ ) eine Monographie „Ueber plötzlichen Tod durch Herzlähmung ${ }^{\prime}$ erscheinen lassen. Eine ausführliche Bearbeitung dieses Gegenstandes ist seitdem wohl nicht erfolgt. Ehrnrooth hätte, ohne mißverstanden zu werden, die Ueberschrift seiner Abhandlung kürzen können, denn der Titel „Ueber plötzlichen Herztod“ hätte damals dasselbe besagt, da die Tatsache, ein plötzlicher Tod könne auch durch Uebererregung des Herzens, wie das bei dem Tod durch Herzkammerflimmern der Fall ist, eintreten, so gut wie unbekannt war. Diese Tatsache ist von ihm auch garnicht in das Bereich seiner Besprechung gezogen worden.

Soweit meine Erfahrung reicht, muß ich sagen, daß mir auf Grund des Tierexperimentes keine Herztodesart bekannt ist, die so rasch zum Tode führen kann wie die durch Herzkammerflimmern. Je rascher also, nach Sekunden bemessen, der beobachtete Herztod beim Menschen erfolgte, desto wahrscheinlicher beruhte er auf Herzkammerflimmern.

Der Tod durch Herzkammerflimmern hat nun, abgesehen von seiner zeitlichen Seite, noch eine ganz besondere Bedeutung.

Das Herzklammerflimmern erklärt nicht nur in

1) In Nr. 14 und 15 der M. m. W. 1912 sprach' ich von Minutentod, gebrauche aber jetzt den Ausdruck Sekundenherztod (s. M. m. W. Nr. 44 S. 1489. 1915), da dieser die Plötzlichkeit noch mehr in den Vordergrund stelit. $-{ }^{2}$ ) Berlin 1904. vielen Fällen die große Plötzlichkeit des Herztodes, sondern läBt überhaupt den Herztod oft erst verstehen, wenn die Sektion keinen für die:Erklärung des Todes hinreichenden Befund ergibt.

Damit komme, ich zu jener Seite des Sekundenherztodes, die ich im Folgenden etwas ausführlicher besprechen möchte, das ist seine anatomische.

Ehrnrooth kommt auf Seite 87 seiner oben erwähnten Abhandlung zu dem Ergebnis: „Der plötzliche Herztod läßt sich durch keine sicheren anatomischen Merkmale erkennen."

- Schon auf S. 3 bemerkt er: „Mit Recht erwähnt Lesser, daß der exakte Beweis dafür, daß der plötzliche Tod als notwendige Konsequenz der gefundenen pathologischen Veränderungen anzusehen ist, nicht immer zu bringen ist", und setzt weiter unten fort: „Wenn wir in manchen Fällen von Herztod die Lähmung des Herzmuskels nicht von den vorgefundenen Veränderungen desselben ableiten können, so müssen wir daran denken, daß die eigentliche Ursache der Herzlähmung außerhalb des Herzens zu suchen ist: im Herznervenzentrum, in den Herznerven, in einer Veränderung des Blutes und überhaupt in fast jedem beliebigen Organ, denn es gibt kein einziges Organ, von dem aus Reflexe auf das Herz nicht möglich wären."

Hierzu wäre Folgendes zu sagen:

Findet der Pathologische Anatom eine Ruptur des Herzens mit Hämoperikard oder eine Embolisierung des Anfangsteiles einer der beiden Koronararterien, so wird er däraus den Schluß ziehen können, daß der Tod plötzlich erfolgt ist, $\cdot$ wenn er auch dahingestellt lassen muß, welchen Zeitraum der Begriff der Plötzlichkeit des Todes umfaßt, was besonders für die Herzruptur gilt, da hierbei auch die Größe der Ruptur von Bedeutung ist.

Bei der Sektion ist es bekanntlich eine Aufgabe des Patho. logischen Anatomen, das anatomische Substrat für den Tod eines Individuums nach Möglichkeit festzustellen. Ob der Tod allmählich oder plötzlich erfolgte, ist eine weitere Frage, die an den Obduzenten nur in gewissen Fällen gestellt wird, und zwar gilt dies wesentlich für gerichtliche Obduktionen; aber auch in diesen Fällen ist es zumeist bekannt, daß das Individuum plötzlich starb, und das Interesse besonders dahingehend, ob der plötzliche Tod ,aus natürlicher Ursache" erfolgte oder ein gewaltsamer Tod anzunehmen ist. Ist es dem Obduzenten aber nicht bekannt, ob es sich um einen plötzlichen Todesfall handelt, dann wird es ihm, soweit es sich um das Herz handelt, nur dann möglich sein, aus dem Herzbefund die Plötzlichkeit des Todes zu erschließen, wenn er anatomische Veränderungen am Herzen findet, wie die oben erwähnten, von denen er weiß, daß sie plötzlich erfolgen. Plötzliche Todesfälle infolge von Herzruptur oder gar infolge Embolisierung einer der beiden Koronararterien sind im Vergleich zu der Zahl der plötzlichen Herztodesfälle Seltenheiten. In den meisten Fällen wird also der Obduzent nicht in der Lage sein, aus dem Herzbefund die Plötzlichkeit des Todes zu erkennen.

$\mathrm{Zu}$ dieser Schwierigkeit gesellt sich noch eine größere. Das ist die Unmöglichkeit, in vielen Fällen aus dem Herzbefund überliaupt den Herztod anatomisch überzeugend begründen zu können.

Dies gilt nicht nur für viele Fälle von Sekundenherztod, sondern auch, wie bekannt und schon oft erörtert worden ist, für viele Fälle weniger plötzlichen, mehr allmählich eintretenden Herztodes. Ja noch mehr, es gilt auch sehr oft von der Herzschwäche, wofür ich hier nur die Untersuchungen und kriti- 
schen Ausführungen von L. Aschoff urd S. Tawaral) allführen will, die zu dem Ergebnis kamen:

„daß die eigenartige Schwäche des hypertrophischen Herzmuskels, soweit unsere bisherigen Erfahrungen reichen, nicht durch den Sitz der Entzündung erklärt werden kann“ und „daß, von Diphtherie a bgesehen, für die überwiegende Zahl aller Herzklappenfehler, Nephritikerherzen etc. die Herzschwäche auch nicht durch den Umfang der a natomischen Läsionen in irgendeinem beachtenswerten Umfang erklärt werden kann. Soweit nicht also das Nervensystem noch eine Rolle bei der Entstehung der Herzschwäehe zu spielen vermag, müssen wir, von den seltenen Fällen ausgedelınter Zerstörung des Reizleitungssystems oder diffuser Myokarditis abgesehen, die wesentliche Ursache derselben in einer funktionellen Schädigung erblicken."

Was meine eigenen Erfanrungen iiber die anatomische Scite des Sekundenherztodes anbelangt, so habe ich, ${ }^{2}$ ) wie mitgeteilt, in der Zeit von 1906-1913 im ganzen acht Fälle beobachtet. In allen diesen Fällen vermochte die Obduktion die Plötzlich keit des Todes nicht zu erklälen, obwohl ein schon makroskopisch deutlicher Herzbefund vorlag. Es handelte sich um Herzklappenfehler, und zwar hauptsächlich um Fehler der Mitralklappen.

In jüngster Zeit hat A. Frank in Köln vier Fälle von Sekundenherztod beobachtet, über die er im Allgemeinen ärztlichen Verein Januar 1916 berichtet hat. Wie ich in der Diskussion $^{3}$ ) hervorhob, unterscheiden sich seine Fälle von meinen wesentlich durch folgende Punkte:

1. durch das Fehlen eines makroskopischen Befundes am Herzen

2. durch die im Verhältnis zu dem erfolgten Tode geringgradigen mikroskopischen Befunde am Herzen,

3. durch das Fehlen klinischer Symptome von seiten des Herzens bei den in dieser Hinsicht sich gesund fühlenden Personen.

Durch diese drei Punkte erschienen mir seine Fälle von besonderer Bedeutung. Abgesehen davon, daß die Plötzlichkeit des Todes durch die Obduktion nicht erklärt wurde, war der Befund am Herzen ein solcher, daß aus ihm der Herztod selbst nicht befriedigend erklärt werden konnte, denn die gefundenen anatomischen Veränderungen am Herzen ließen höchstens annehmen, daß in vivo ein gewisser Grad von Herzschwäche bestand.

Nun war aber von Symptomen einer Herzschwäche in jenen Fällen nichts bekannt; während der Arbeit fielen einige der obduzierten Männer tot um. Wären vielleicht auch bei genauer klinischer Untersuchung Herzsymptome gefunden worden, so ist doch zu betonen, daß die Gestorbenen sich in dieser Hinsicht nicht krank fühlten, wenigstens sich nicht genötigt sahen, zum Arzte zu gehen, vielmehr ihrer Arbeit weiter nachgingen.

Daraus folgt, daß, wenn auch der anatomische Befund einen gewissen Grad von Herzschwäche annehmen ließ, dieser Grad nach dem Verhalten der Gestorbenen in vivo relativ gering gewesen sein wird.

Wenn wir uns nun fragen, aut welche Weise in diesen Fällen der Tod zustandekam, so ist es wichtig zu betonen, daß wir uns hüten müssen, bei Beantwortung dieser Frage einen einseitigen Standpunkt einzunehmen, wie dies nicht selten geschieht, besonders im sogenannten praktischen Leben, wo wir im abgekürzten Verfahren nach, ,der Todesursache" fragen. Der Tod tritt immer als die Wirkung einer Anzahl von Ursachen ein. Von diesen wird jedoch gewöhnlich eine je nach dem jeweiligen Standpunkt und Bedürfnis besonders hervorgehoben. Tun wir dies unter dem Gebrauch des Ausdruckes Koëffizien t ${ }^{4}$ ) für Uisache, so werden wir nicht in den Fehler verfallen, in den wir bei der Frage nach ,der Todesursache" nur zu leicht geraten, nämlich zu glauben, der Tod könne lediglich infolge einer Ursache eintreten, denn Koëffizient bedeutet soviel als das Mitwirkende; so erinnert diese Bezeichnungsweise von selbst daran, daß kein Vorgang durch eine Ursache allein hervorgerufen wird.

Wie bei einer Erkrankung, so können wir auch beim Tod, allgemein gefaßt, zwei Koëffizienten unterscheiden, seinen dis ponierenden und seinen auslösenden. Wenden wir diese

1) Die heutige Lehre von den pathologisch-anatomischen Grundlagen der Herzschwäche. Jena 1906. ${ }^{2}{ }^{2}$ ) M. m. W. 1912 Nr. 14 u. 15 und Prag. m. W. 1913 Nr. 38. - ${ }^{3}$ ) M. m. W. 1916 Nr. 11 S. 393. 4) Die Naturwissensehaften 1913 H. 7.
Erkenntnis auf den Sekundenherztod an, so können wir sagen, daß er zwar immer die Wirkung beider Koëffizienten ist, daß aber der Anteil, welcher der eine oder der andere an ihm hat, sehr verschieden sein kann. Diesen Anteil können wir nun in Wirklichkeit nicht zahlenmäßig angeben, sondern nur zum Teil, und dann auch nur sehr beiläufig abschätzen. Am besten ist dies noch möglich, wenn der Koêffizient materieller Natur ist, während er sich einer Messung gänzlich entzieht, wenn es ein psychischer, z. B. eine erschreckende Nachricht ist. So ist es begreiflich, daß auch in dieser Hinsicht der Koëffizient mit anatomischem Substrat - nennen wir ihn den an atomischen Koëffizienten - dem funktionellen. Koëffizienten im allgemeinen den Rang abläuft und nicht selten überschätzt wird, denn die anatomische Veränderung läßt sich zahlenmäBig ungefähr abschätzen, die funktionelle gewöhnlich nicht, ganz abgesehen davon, daß die erstere post mortem bestehen bleibt, die letztere vorübergeht. Ist nun gar der funktionelle Koëffizient ein so plötzlich einwirkendel und tritt das Versagen von Herzund Atemtätigkeit so rasch ein, wie beim Sekundenherztode, dann ist auch die Möglichleit, sich über sein Zustandekommen in den einzelnen Fällen Aufschluß zu verschaffen, für den Arzt meistens kaum möglich, und er ist sehr oft nur auf Vermutungen angewiesen.

Auch dem Obduzenten fehlt, wie wir sahen, in den meisten Fällen zur ergänzenden Erklärung seines Sektionsbefundes die Kenntnis des funktionellen Koëffizienten; er ist aber in der glücklichen Lage, wenigstens den anatomischen Koëffizienten und damit den disponierenden, soweit er sich anatomisch feststellen läßt, zumeist ausfindig machen zu können. Es ist daher verständlich, daß wir über den disponierenden Koëffizienten des Sekundenherztodes in so mancher Hinsicht besser unterrichtet sind als über den auslösenden. Aber selbst wenn vom Arzt beobachtet wurde, daß ein Mensch im unmittelbaren Anschluß z. B. an eine erschreckende Mitteilung einen Sekundenherztod erlitt, und wenn sich auch bei der Obduktion Verände. rungen am Herzen fanden und damit das Organ und die Art seiner Erkrankung festgestellt wird, mit der der Tod in $\mathrm{Zu}$ sammenhang steht, so blieb doch in vielen Fällen unbeant. wortet, wie das Zusammenwirken des in der Form anatomischer Veränderungen an diesem Organe festgestellten disponierenden Koëffizienten mit dem auslösenden Koëffizienten den Sekundenherztod herbeigeführt hat.

Eine Antwort hat erst das Tierexperiment gegeben, indem es den Arzt mit einer Art plötzlichen Versagens des Herzens bekannt machte, dem Herzkammerflimmern, das beim Exp rriment sehr häufig den Tod des Versuchstieres herbeiführt. Diese Herztodesart erklärt, wie eingangs schon erwähnt, nicht nur in vielen Fällen von Herztod seine Plötzlichkeit, sondern macht es auch verständlich, daß in p!ötzlichen Herztodesfällen der anatomische Befund oft relativ geringgradig ist.

Auf diese Weise lassen sich viele Fälle von Sekundenherztod beim Status thymico-lymphaticus, ${ }^{1}$ ) bei Angina pectoris, ${ }^{2}$ ) bei der Chloroformnarkose ${ }^{3}$ ) etc. verstehen.

An dieser Stelle ist es notwendig, auf die Bemühungen von J. H. Mönckeberg ${ }^{4}$ ) hinzuweisen, Fälle von plötzlichem Herztod durch eine totale Aufhebung der Funktionen des Atrioventrikularsystems zu erklären, also durch eine ganz andere Herztodesart. Er wies darauf hin, daß in vielen Fällen von Dissoziation oder Kammersystolenausfall eine überraschend große Prozentzahl von Angaben über plötzlichen Herztod sich findet. Es ist gewiß wichtig, daß der Adams-Stokessche Symptomenkomplex öfters zu plötzlichem Tode führt. Leider sind aber die klnischen Angaben hinsichtlich der Plötzlichkeit, mit der der Tod eintrat, zumeist nicht scharf genug, um daraus zu ersehen, ob es sich um einen Sekundenherztod handelte. In der plötzlichen Aufhebung der Ueberleitung hätte man anscheinend den plötzlich einsetzenden Koëffizienten. Daß jedoch die plötzliche Aufhebung der Ueberleitung den Tod herbeigeführt hätte, darüber ist weder klinisch etwas Sicheres bekannt, noch ist es mir experimentell jemals vorgekommen. Auch ließe er sich nur in solchen Fällen verstehen, in denen die Reizbildung

1) M. m. W. 1912 Nr. 14 u. 15. - 2) M. m. W. 1915 Nr. 44. $\left.{ }^{3}\right)$ M. m. W. 1916. - ${ }^{4}$ ) Untersuchungen über das Atrioventrikularbündel im menschlichen Herzen, Jena 1908; Ergebnisse der Allg. Path. u. Path. Anat. 14. 1910 S. 534. 
in den Kammern nach Aufhebung der Ueberleitung sich nicht entwickeln würde, wozu eine sehr hochgradige Schädigung der Kammern erforderlich wäre. Bei schon bestehender Dissoziation, also bei Kammerautomatie, den plötzlichen Herztod auf ein plötzliches Versagen der Reizbildung zu beziehen, hätte man theoretisch insofern anscheinend Anlaß, als es bei Kammerautomatie zu hochgradiger Kammerbradysystolie kommen kann. Indessen ist bis jetzt das plötzliche Aufhören der Kammertätigkeit durch plötzliches Versagen der Kammerreizbildung weder klinisch nachgewiesen, noch experimentell mir begegnet. Soweit es sich in den Fällen von Adams-Stokesschem Symptomenkomplex um einen Sekundenherztod handelt, ist nach meiner Meinung der Tod sehr wahrscheinlich durch Herzkammerflimmern herbeigeführt. Insofern es sich um Erkrankungen des Artrioventrikularsystems handelt, kann man in diesen, besonders soweit es sich um mehr akut einsetzende Veränderungen handelt, wohl einen Koëffizienten des Sekundenherztodes erblicken, aber nicht im Sinne der Aufhebung der Funktionen dieses Systems, sondern vielmehr im Sinne einer plötzlich einsetzenden Uebererregung, ausgehend von irgendeiner Stelle des Systems.

Im Anschluß an diese kurze Erörterung der Beziehung des Atrioventrikularsystems zu plötzlichen Herztodesfällen möchte ich hervorheben, daß die Veränderungen, die A. Frank in seinen oben erwähnten Fällen fand, nach seiner Angabe ihren Sitz nicht in den Hauptabschnitten des Reizleitungssystems hatten.

Was die klin is che Seite des Sekundenherztodes anbelangt, so ist es klar, daß die Kenntnis solcher Fälle für den praktischen Arzt von großer Wichtigkeit ist. Immer wieder werden sie darauf hinweisen, bei der Prognose der Herzerkrankungen quoad vitam sehr vorsichtig zu sein.

Beim Vorhandensein gewisser, eine nur geringgradige Herzschwäche anzeigender Herzsymptome (von denen die objektiven oft nicht einmal sicher nachweisbar sind) kann das Ende sozusagen mit einem Sprunge erreicht werden. In den Fällen von Frank war über Herzsymptome, wie erwähnt, nichts bekannt. $\mathrm{Da}$ die Personen jedoch während der Arbeit anscheinend aus voller Gesundheit tot umfielen, ist wohl der Schluß berechtigt, daß die betreffenden Männer wenigstens keine solche Herżsymptome hatten, daß sie durch letztere genötigt worden wären, zum Arzte zu gehen. Nach dem histologischen Befund gehören diese Fälle vielleicht in die Klasse der Rekonvaleszenten nach Infektionskrankheiten, von denen man schon seit langer Zeit weiß, daß sie ein labiles Herz besitzen.

Bei einem der Fälle trat der Sekundenherztod ein, als der auf einem Stuhle sitzende Mann vom Arzte an einem Arme mit schwachen Strömen elektrisiert wurde. In diesem Falle konnte der Arzt auch die ganz plötzlich eingetretene Pulslosigkeit feststellen, jenes Symptom, das, wie ich ${ }^{1}$ ) seinerzeit schon hervorgehoben habe, die Diagnose wesentlich stützt, daß der Tod durch Herzkammerflimmern eingetreten ist, denn bei letzterem verschwindet sofort der Puls, ist eine Kammertätigkeit nur noch mit Hilfe des Elektrokardiographen, und zwar in Form zahlreicher kleiner Oszillationen, nachweisbar und pflegt die Atmung die Herztätigkeit für kurze Zeit zu überdauern.

Der Sekundenherztod spielt ferner bei der Begutachtung von Unfalltodesfällen und bei der Beurteilung gerichtlich-medizinischer Fälle eine bedeutsame Rolle, zwei Punkte, auf die hier nur ganz kurz hingewiesen werden kann. Es sei nur erwähnt, daß speziell die gerichtliche Medizin seit jeher viel Anlaß hatte, sich bei den plötzlichen Todesfällen aus natürlicher Ursache auch mit dem plötzlichen Herztod zu befassen, und so finden wir über letzteren viel in den Büchern der gerichtlichen Medizin $^{2}$ ), während in den pathologisch-anatomischen Lehrbüchern ${ }^{3}$ ) darüber recht wenig steht. Letzteres wird aus dem verständlich, was wir weiter oben über die Erkennung der Plötzlichkeit und des Herztodes selbst aus dem anatomischen Herzbefund angeführt haben. Soweit es sich um den Grad der Plötzlichkeit des Todes und seine ganze feststellbare Vorgeschichte

1) M. m. W. 1912 Nr. 14 u. 15. - $\left.{ }^{2}\right)$ Sehe z. B. Handbuch der ärztl. Sachverständigen-Tätigkeit II. Piötzlicher Tod aus natïrlicher Ursache, A. Kolisko, 1913. - 3) Siehe z. B. Pathologische Anatomie, herausgegeb. von L. As chof f, 3. Aufl. 1913. handelt, fällt der Sekundenherztod in das Bereich des Arztes; soweit es seine anatomisch feststellbaren Grundlagen betrifft, gehört er in das Gebiet des Pathologischen Anatomen. Durch ihr Zusammenwirken, wie wir es besonders ausgebildet in der gerichtlichen Medizin finden, erfahren die Fälle von plötzlichem Herztod ihre Aufklärung, soweit dies bisher möglich war. Für so manche als Sekundenherztod zeitlich gekennzeichneten Fälle bringt nun die Pathologische Physiologie die fehlende weitere Aufklärung. So sehen wir wieder, wie die Vereinigung von klinischer Beobachtung, anatomischer Untersuchung und experimenteller Forschung uns fortschreiten läßt auf den schwierigen Wegen medizinischer Erkenntnis und daß auch letztere das Ergebnis einer Pluralität von Koëffizienten ist. 